International Journal of Pure and Applied Mathematics

Volume 99 No. 3 2015, 343-354

ISSN: 1311-8080 (printed version); ISSN: 1314-3395 (on-line version)

url: http://www.ijpam.eu

doi: http://dx.doi.org/10.12732/ijpam.v99i3.9

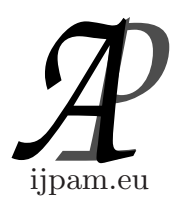

\title{
CONSTRUCTION OF TCHEBYSHEV-II WEIGHTED ORTHOGONAL POLYNOMIALS ON TRIANGULAR
}

\author{
Mohammad A. AlQudah \\ Department of Mathematics \\ Northwood University \\ Midland, MI 48640 USA
}

\begin{abstract}
We construct Tchebyshev-II (second kind) weighted orthogonal polynomials $\mathscr{U}_{n, r}^{(\gamma)}(u, v, w), \gamma>-1$, on the triangular domain $T$. We show that $\mathscr{U}_{n, r}^{(\gamma)}(u, v, w), n=0,1,2, \ldots, r=0,1, \ldots, n$, form an orthogonal system over $T$ with respect to the Tchebyshev-II weight function.
\end{abstract}

AMS Subject Classification: 42C05, 33C45, 33C70

Key Words: Tchebyshev, orthogonal, triangular, Bernstein, bivariate

\section{Introduction}

In the last couple decades, orthogonal polynomials have been studied thoroughly [8] and [12]. The Tchebyshev orthogonal polynomial of the second kind (Tchebyshev-II) is among these orthogonal polynomials. Although the main definitions and basic properties were defined many years ago, see [3] and [11], the cases of bivariate or more variables are limited.

Tchebyshev-II polynomials $U_{n, r}^{(\gamma)}(u, v, w)$ are orthogonal to each polynomial of degree $\leq n-1$, with respect to the weight function $\mathrm{W}^{(\gamma)}(u, v, w)=u^{\frac{1}{2}} v^{\frac{1}{2}}(1-$ $w)^{\gamma}, \gamma>-1$ on triangular domain $T$ as defined in [1] and [8]. However, for $r \neq s, U_{n, r}^{(\gamma)}(u, v, w)$ and $U_{n, s}^{(\gamma)}(u, v, w)$ are not orthogonal with respect to the weight function $\mathrm{W}^{(\gamma)}(u, v, w)$ on $T$.

Received: November 18, 2014

(c) 2015 Academic Publications, Ltd. url: www.acadpubl.eu 
In this paper, we construct bivariate orthogonal polynomials $\mathscr{U}_{n, r}^{(\gamma)}(u, v, w)$, $r=0,1, \ldots, n ; n=0,1,2, \ldots$, with respect to the Tchebyshev-II weight function $\mathrm{W}^{(\gamma)}(u, v, w)=u^{\frac{1}{2}} v^{\frac{1}{2}}(1-w)^{\gamma}, \gamma>-1$, on triangular domain $T$. We show that $\mathscr{U}_{n, r}^{(\gamma)}(u, v, w)$ form an orthogonal system over the triangular domain $T$ with respect to the weight function $\mathrm{W}^{(\gamma)}(u, v, w)=u^{\frac{1}{2}} v^{\frac{1}{2}}(1-w)^{\gamma}, \gamma>-1$. Worth to mention that these Tchebyshev-II weighted orthogonal polynomials are given in the Bernstein basis form; they preserve many geometric properties of the Bernstein polynomial basis.

The construction of bivariate orthogonal polynomials on the square $G$ is straightforward [11], where $G=\{(x, y):-1 \leq x \leq 1,-1 \leq y \leq 1\}$. It can be done by considering the tensor product of the set of orthogonal polynomials over $G$.

Let $\left\{U_{n}(x)\right\}$ be the Tchebyshev-II polynomials over $[-1,1]$ with respect to the weight function $\mathrm{W}_{1}(x)=\left(1-x^{2}\right)^{\frac{1}{2}}$, and $\left\{Q_{m}(y)\right\}$ be the Tchebyshev-II polynomials over $[-1,1]$ with respect to the weight function $\mathrm{W}_{2}(y)=\left(1-y^{2}\right)^{\frac{1}{2}}$. The bivariate polynomials $\left\{R_{n m}(x, y)\right\}$ on $G$ formed by the tensor products of the Tchebyshev-II polynomials defined as

$$
R_{n m}(x, y):=U_{n-m}(x) Q_{m}(y), n=0,1,2, \ldots, m=0,1, \ldots, n .
$$

The bivariate polynomials $\left\{R_{n m}(x, y)\right\}$ are orthogonal on the square $\mathrm{G}$ with respect to the weight function $\mathrm{W}(x, y)=\mathrm{W}_{1}(x) \mathrm{W}_{2}(y)$. However, the construction of orthogonal polynomials over a triangular domains are not straightforward like the tensor product over the square $G$.

\section{Bernstein and Orthogonal Polynomials over Triangular Domains}

Consider a base triangle in the plane with the vertices $\mathbf{p}_{k}=\left(x_{k}, y_{k}\right), k=1,2,3$. Then every point $\mathbf{p}$ inside the triangle $T$ can be written using the barycentric coordinates $(u, v, w)$ as $\mathbf{p}=u \mathbf{p}_{1}+v \mathbf{p}_{2}+w \mathbf{p}_{3}$, where $u, v, w \geq 0, u+v+w=1$. The barycentric coordinates are the ratio of areas of subtriangles of the base triangle as follows:

$$
u=\frac{\operatorname{area}\left(\mathbf{p}, \mathbf{p}_{2}, \mathbf{p}_{3}\right)}{\operatorname{area}\left(\mathbf{p}_{1}, \mathbf{p}_{2}, \mathbf{p}_{3}\right)}, \quad v=\frac{\operatorname{area}\left(\mathbf{p}_{1}, \mathbf{p}, \mathbf{p}_{3}\right)}{\operatorname{area}\left(\mathbf{p}_{1}, \mathbf{p}_{2}, \mathbf{p}_{3}\right)}, \quad w=\frac{\operatorname{area}\left(\mathbf{p}_{1}, \mathbf{p}_{2}, \mathbf{p}\right)}{\operatorname{area}\left(\mathbf{p}_{1}, \mathbf{p}_{2}, \mathbf{p}_{3}\right)} .
$$

where $\mathbf{p}_{1}, \mathbf{p}_{2}, \mathbf{p}_{3}$ are not collinear. 
Definition 2.1. The Bernstein polynomials $b_{i}^{n}(u), u \in[0,1], i=0,1, \ldots, n$, are defined by:

$$
b_{i}^{n}(u)= \begin{cases}\left(\begin{array}{l}
n \\
i
\end{array}\right) u^{i}(1-u)^{n-i} & i=0,1, \ldots, n \\
0 & \text { else }\end{cases}
$$

where $\left(\begin{array}{l}n \\ i\end{array}\right)$ are the binomial coefficients.

Let $\zeta=(i, j, k)$ denote triples of nonnegative integers, where $|\zeta|=i+j+k$. The generalized Bernstein polynomials of degree $n$ on the triangular domain

$$
T=\{(u, v, w): u, v, w \geq 0, u+v+w=1\},
$$

are defined by

$$
b_{\zeta}^{n}(u, v, w)=\left(\begin{array}{l}
n \\
\zeta
\end{array}\right) u^{i} v^{j} w^{k}, \text { where }|\zeta|=n \text { and }\left(\begin{array}{l}
n \\
\zeta
\end{array}\right)=\frac{n !}{i ! j ! k !} .
$$

Note that the generalized Bernstein polynomials are nonnegative over $T$ and form a partition of unity,

$$
1=(u+v+w)^{n}=\sum_{\substack{0 \leq i, j, k \leq n \\ i+j+k=n}} \frac{n !}{i ! j ! k !} u^{i} v^{j} w^{k} .
$$

These polynomials define the Bernstein basis for the space $\Pi_{n}$, the space of all polynomials of degree $n$ over the triangular domain $T$.

A basis of linearly independent and mutually orthogonal polynomials in the barycentric coordinates $(u, v, w)$ are constructed over $T$. These polynomials are $\begin{array}{rr}b_{0,0} & \\ b_{1,0} & b_{1,1}\end{array}$

represented in the following triangular table $\begin{array}{llll}b_{2,0} & b_{2,1} & b_{2,2}\end{array}$

$$
b_{n, 0} \quad b_{n, 1} \quad b_{n, 2} \quad \ldots \quad b_{n, n} .
$$

The $k$ th row of this table contains $k+1$ polynomials. Thus, there are $\frac{(n+1)(n+2)}{2}$ polynomials in a basis of linearly independent polynomials of total degree $n$. Therefore, the sum (3) involves a total of $\frac{(n+1)(n+2)}{2}$ linearly independent polynomials.

Any polynomial $p(u, v, w)$ of degree $n$ can be written in the Bernstein form

$$
p(u, v, w)=\sum_{|\zeta|=n} d_{\zeta} b_{\zeta}^{n}(u, v, w)
$$


with Bézier coefficients $d_{\zeta}$. We can use the degree elevation algorithm for the Bernstein representation (4) by multiplying both sides by $1=u+v+w$ and writing

$$
p(u, v, w)=\sum_{|\zeta|=n+1} d_{\zeta}^{(1)} b_{\zeta}^{n+1}(u, v, w)
$$

where the the coefficients $d_{\zeta}^{(1)}$ are defined in [4] and [7] as

$$
d_{i, j, k}^{(1)}=\frac{1}{n+1}\left(i d_{i-1, j, k}+j d_{i, j-1, k}+k d_{i, j, k-1}\right), \quad i+j+k=n+1 .
$$

Lemma 2.1. [5] The Bernstein polynomials $b_{\zeta}^{n}(u, v, w),|\zeta|=n$, on $T$ satisfy

$$
\iint_{T} b_{\zeta}^{n}(u, v, w) d A=\frac{\Delta}{(n+1)(n+2)},
$$

where $\Delta$ is double the area of $T$.

Definition 2.2. Let $p(u, v, w)$ and $q(u, v, w)$ be two bivariate polynomials over $T$, then their inner product over $T$ defined by

$$
\langle p, q\rangle=\frac{1}{\Delta} \iint_{T} p q d A, \text { where } p \text { and } q \text { are orthogonal if }\langle p, q\rangle=0 .
$$

For $m \geq 1, \mathfrak{L}_{\mathfrak{m}}=\left\{p \in \Pi_{m}: p \perp \Pi_{m-1}\right\}$ is the space of polynomials of degree $m$ that are orthogonal to all polynomials of degree $<m$ over a triangular domain $T$.

Let $f(u, v, w)$ be an integrable function over $T$ and consider the operator

$$
S_{n}(f)=(n+1)(n+2) \sum_{|\zeta|=n}\left\langle f, b_{\zeta}^{n}\right\rangle b_{\zeta}^{n}
$$

For $n \geq m, \lambda_{m, n}=\frac{(n+2) ! n !}{(n+m+2) !(n-m) !}$ is an eigenvalue of the operator $S_{n}$, and $\mathfrak{L}_{\mathfrak{m}}$ is the corresponding eigenspace [2]. The following two lemmas will be used in the proof of the main results.

Lemma 2.2. [5] Let $p=\sum_{|\zeta|=n} c_{\zeta} b_{\zeta}^{n} \in \mathfrak{L}_{\mathfrak{m}}$ and let $q=\sum_{|\zeta|=n} d_{\zeta} b_{\zeta}^{n} \in \Pi_{n}$ with $m \leq n$. Then,

$$
\langle p, q\rangle=\frac{(n !)^{2}}{(n+m+2) !(n-m) !} \sum_{|\zeta|=n} c_{\zeta} d_{\zeta} .
$$


Lemma 2.3. [5] Let $p=\sum_{|\zeta|=n} c_{\zeta} b_{\zeta}^{n} \in \Pi_{n}$. Then,

$$
p \in \mathfrak{L}_{\mathfrak{n}} \Longleftrightarrow \sum_{|\zeta|=n} c_{\zeta} d_{\zeta}=0 \quad \forall q=\sum_{|\zeta|=n} d_{\zeta} b_{\zeta}^{n} \in \Pi_{n-1}
$$

For the main results simplifications, we define the double factorial of an integer $n$ as

$$
\begin{aligned}
(2 n-1) ! ! & =(2 n-1)(2 n-3)(2 n-5) \ldots(3)(1) & & \text { if } n \text { is odd } \\
n ! ! & =(n)(n-2)(n-4) \ldots(4)(2) & & \text { if } n \text { is even }
\end{aligned}
$$

where $0 ! !=(-1) ! !=1$.

\section{Tchebyshev-II Weighted Orthogonal Polynomials}

Tchebyshev-II polynomials $U_{n}(x)$ of degree $n$ are the orthogonal polynomials except for a constant factor on $[-1,1]$ with respect to the weight function $\mathrm{W}(x)=\left(1-x^{2}\right)^{\frac{1}{2}}$. For simplicity, without loss of generality, we take $x \in[0,1]$ for both Bernstein and Tchebyshev-II polynomials.

The following lemmas will be needed in the construction of the orthogonal bivariate polynomials and the proof of the main results.

Lemma 3.1. [10] The Tchebyshev-II polynomials $U_{r}(x)$ have the Bernstein representation:

$$
U_{r}(x)=\frac{(r+1)(2 r) ! !}{(2 r+1) ! !} \sum_{i=0}^{r}(-1)^{r-i} \frac{\left(\begin{array}{c}
r+\frac{1}{2} \\
i
\end{array}\right)\left(\begin{array}{c}
r+\frac{1}{2} \\
r-i
\end{array}\right)}{\left(\begin{array}{c}
r \\
i
\end{array}\right)} b_{i}^{r}(x), r=0,1, \ldots
$$

Lemma 3.2. [10] The Tchebyshev-II polynomials $U_{0}(x), \ldots, U_{n}(x)$ of degree $\leq n$ can be expressed in the Bernstein basis of fixed degree $n$ by the following formula

$$
U_{r}(x)=\sum_{i=0}^{n} \mu_{i, r}^{n} b_{i}^{n}(x), \quad r=0,1, \ldots, n
$$

where

$$
\mu_{i, r}^{n}=\frac{(r+1)(2 r) ! !}{(2 r+1) ! !}\left(\begin{array}{c}
n \\
i
\end{array}\right)^{-1} \sum_{k=\max (0, i+r-n)}^{\min (i, r)}(-1)^{r-k}\left(\begin{array}{c}
n-r \\
i-k
\end{array}\right)\left(\begin{array}{c}
r+\frac{1}{2} \\
k
\end{array}\right)\left(\begin{array}{c}
r+\frac{1}{2} \\
r-k
\end{array}\right)
$$


Using Pochhammer symbol is more appropriate in (3.1) and (8), but the combinatorial notation gives more compact and readable formulas, these have also been used by Szegö [12].

In the following lemma, let

$$
q_{n, r}(w)=\sum_{j=0}^{n-r}(-1)^{j}\left(\begin{array}{c}
n+r+1 \\
j
\end{array}\right) b_{j}^{n-r}(w) .
$$

The polynomial $q_{n, r}(w)$ is a scalar multiple of $U_{n-r}(1-2 w)$.

Lemma 3.3. [5] For $r=0, \ldots, n$ and $i=0, \ldots, n-r-1, q_{n, r}(w)$ is orthogonal to $(1-w)^{2 r+i+1}$ on $[0,1]$. Hence for every polynomial $p(w)$ of degree $\leq n-r-1$, we have

$$
\int_{0}^{1} q_{n, r}(w) p(w)(1-w)^{2 r+1} d w=0
$$

Analogous to [5], a simple closed-form representation of degree-ordered system of orthogonal polynomials is constructed on a triangular domain $T$ using Bernstein polynomials, since Bernstein polynomials are stable [6].

For $r=0,1, \ldots, n$ and $n=0,1,2, \ldots$, we define the bivariate polynomials

$$
\mathscr{U}_{n, r}^{(\gamma)}(u, v, w)=\sum_{i=0}^{r} c(i) b_{i}^{r}(u, v) \sum_{j=0}^{n-r}(-1)^{j}\left(\begin{array}{c}
n+r+1 \\
j
\end{array}\right) b_{j}^{n-r}(w, u+v),
$$

where $\gamma>-1, b_{i}^{r}(u, v)$ defined in $(2)$ and

$$
c(i)=(-1)^{r-i} \frac{\left(\begin{array}{c}
r+\frac{1}{2} \\
i
\end{array}\right)\left(\begin{array}{c}
r+\frac{1}{2} \\
r-i
\end{array}\right)}{\left(\begin{array}{c}
r \\
i
\end{array}\right)}, \quad i=0,1, \ldots, r .
$$

By choosing $\mathscr{U}_{0,0}^{(\gamma)}=1$, the polynomials $\mathscr{U}_{n, r}^{(\gamma)}(u, v, w)$ for $0 \leq r \leq n$ and $n=0,1,2, \ldots$ form a degree-ordered orthogonal sequence over $T$.

Rewriting (10) using Tchebyshev-II polynomials form, we obtain

$$
\mathscr{U}_{n, r}^{(\gamma)}(u, v, w)=\sum_{i=0}^{r} c(i) \frac{b_{i}^{r}(u, v)}{(u+v)^{r}}(1-w)^{r} \sum_{j=0}^{n-r}(-1)^{j}\left(\begin{array}{c}
n+r+1 \\
j
\end{array}\right) b_{j}^{n-r}(w, 1-w)
$$

Using Lemma 3.1 and $\frac{b_{i}^{r}(u, v)}{(u+v)^{r}}=b_{i}^{r}\left(\frac{u}{1-w}\right)$, and we get

$$
\mathscr{U}_{n, r}^{(\gamma)}(u, v, w)=\frac{\left(\begin{array}{c}
r+\frac{1}{2} \\
r
\end{array}\right)}{(r+1)} U_{r}\left(\frac{u}{1-w}\right)(1-w)^{r} q_{n, r}(w), \quad r=0, \ldots, n
$$


where $U_{r}(t)$ is the univariate Tchebyshev-II polynomial of degree $r$ and $q_{n, r}(w)$ is defined in equation (9).

For simplicity, we rewrite (12) as

$$
\mathscr{U}_{n, r}^{(\gamma)}(u, v, w)=U_{r}\left(\frac{u}{1-w}\right)(1-w)^{r} q_{n, r}(w), \quad r=0, \ldots, n,
$$

since we are dealing with orthogonality, and the Tchebyshev-II polynomials $U_{n}(x)$ of degree $n$ are the orthogonal except for a constant factor.

The polynomials $\mathscr{U}_{n, r}^{(\gamma)}(u, v, w)$ form an orthogonal system if $\mathscr{U}_{n, r}^{(\gamma)}(u, v, w) \in$ $\mathfrak{L}_{\mathfrak{n}}, n \geq 1, r=0,1, \ldots, n$, and for $r \neq s \mathscr{U}_{n, r}^{(\gamma)}(u, v, w) \perp \mathscr{U}_{n, s}^{(\gamma)}(u, v, w)$. In the following theorem, we show that the polynomials $\mathscr{U}_{n, r}^{(\gamma)}(u, v, w), r=0, \ldots, n$, are orthogonal to all polynomials of degree less than $n$ over the triangular domain $\mathrm{T}$.

Theorem 3.1. For each $r=0,1, \ldots, n$ and $n=1,2, \ldots, \mathscr{U}_{n, r}^{(\gamma)}(u, v, w) \in$ $\mathfrak{L}_{n}$ with respect to the weight function $\mathrm{W}^{(\gamma)}(u, v, w)=u^{\frac{1}{2}} v^{\frac{1}{2}}(1-w)^{\gamma}$, where $\gamma>-1$.

Proof. Let

$$
Q_{s, m}(u, v, w)=U_{s}\left(\frac{u}{1-w}\right)(1-w)^{m} w^{n-m-1}, m=0, \ldots, n-1, s=0, \ldots, m,
$$

be the set of bivariate polynomials. The span of (14) includes the set of Bernstein polynomials

$$
\begin{aligned}
b_{j}^{m}\left(\frac{u}{1-w}\right)(1-w)^{m} w^{n-m-1} & =b_{j}^{m}(u, v)(1-w)^{m} w^{n-m-1} \frac{1}{(1-w)^{m}} \\
& =b_{j}^{m}(u, v) w^{n-m-1}, \quad j=0, \ldots, m ; m=0, \ldots, n-1,
\end{aligned}
$$

which span $\Pi_{n-1}$.

It is sufficient to show that for each $s=0, \ldots, m ; m=0, \ldots, n-1$,

$$
I:=\iint_{T} \mathscr{U}_{n, r}^{(\gamma)}(u, v, w) Q_{s, m}(u, v, w) \mathrm{W}^{(\gamma)}(u, v, w) d A=0 .
$$

The integral (15) can be simplified to

$$
I=\Delta \int_{0}^{1} \int_{0}^{1-w} U_{r}\left(\frac{u}{1-w}\right) q_{n, r}(w) U_{s}\left(\frac{u}{1-w}\right) w^{n-m-1} u^{\frac{1}{2}} v^{\frac{1}{2}}(1-w)^{\gamma+r+m} d u d w .
$$


Using the substitution $t=\frac{u}{1-w}$ in (16) we have

$$
\begin{aligned}
I & =\Delta \int_{0}^{1} \int_{0}^{1} U_{r}(t) q_{n, r}(w) U_{s}(t)(1-w)^{\gamma+r+m+2} w^{n-m-1} t^{\frac{1}{2}}(1-t)^{\frac{1}{2}} d t d w \\
& =\Delta \int_{0}^{1} U_{r}(t) U_{s}(t) t^{\frac{1}{2}}(1-t)^{\frac{1}{2}} d t \int_{0}^{1} q_{n, r}(w)(1-w)^{\gamma+r+m+2} w^{n-m-1} d w .
\end{aligned}
$$

If $m<r$, then $s<r$, the first integral is zero by the orthogonality property of the Tchebyshev-II polynomials. If $r \leq m \leq n-1$, then by Lemma 3.3 the second integral equals zero. Thus the theorem follows.

Note that taking $\mathrm{W}^{(\gamma)}(u, v, w)=u^{\frac{1}{2}} v^{\frac{1}{2}}(1-w)^{\gamma}$ enables us to separate the integrand in the proof of Theorem 3.1. Also taking $\gamma>-1$ enables us to use Lemma 3.3 in the proof of Theorem 3.1.

In the following theorem, we show that $\mathscr{U}_{n, r}^{(\gamma)}(u, v, w)$ is orthogonal to each polynomial of degree $n$.

Theorem 3.2. For $r \neq s, \mathscr{U}_{n, r}^{(\gamma)}(u, v, w) \perp \mathscr{U}_{n, s}^{(\gamma)}(u, v, w)$ with respect to the weight function $\mathrm{W}^{(\gamma)}(u, v, w)=u^{\frac{1}{2}} v^{\frac{1}{2}}(1-w)^{\gamma}$ where $\gamma>-1$.

Proof. For $r \neq s$, we have

$$
\begin{aligned}
I:= & \iint_{T} \mathscr{U}_{n, r}^{(\gamma)}(u, v, w) \mathscr{U}_{n, s}^{(\gamma)}(u, v, w) \mathrm{W}^{(\gamma)}(u, v, w) d A \\
= & \Delta \int_{0}^{1} \int_{0}^{1-w} U_{r}\left(\frac{u}{1-w}\right) U_{s}\left(\frac{u}{1-w}\right)(1-w)^{r+s} q_{n, r}(w) q_{n, s}(w) \\
& \mathrm{W}^{(\gamma)}(u, v, w) d u d w .
\end{aligned}
$$

Using the substitution $t=\frac{u}{1-w}$, we get

$$
I=\Delta \int_{0}^{1} U_{r}(t) U_{s}(t) t^{\frac{1}{2}}(1-t)^{\frac{1}{2}} d t \int_{0}^{1} q_{n, r}(w) q_{n, s}(w)(1-w)^{\gamma+r+s+2} d w .
$$

the first integral equals zero by orthogonality property of the Tchebyshev-II polynomials for $r \neq s$, and thus the theorem follows. 


\section{Orthogonal Polynomials in Bernstein Basis}

The Bernstein-Bézier form of curves and surfaces exhibits some interesting geometric properties, see [4] and [7]. Writing the orthogonal polynomials $\mathscr{U}_{n, r}^{(\gamma)}(u, v$, $w), r=0,1, \ldots, n$ and $n=0,1,2, \ldots$ in the following Bernstein-Bézier form:

$$
\mathscr{U}_{n, r}^{(\gamma)}(u, v, w)=\sum_{|\zeta|=n} a_{\zeta}^{n, r} b_{\zeta}^{n}(u, v, w)
$$

The following theorem provides a closed form of the Bernstein coefficients $a_{\zeta}^{n, r}$

Theorem 4.1. The Bernstein coefficients $a_{\zeta}^{n, r}$ are given by

$$
a_{i j k}^{n, r}=\left\{\begin{array}{ll}
(-1)^{k} \frac{\left(\begin{array}{c}
n+r+1 \\
k
\end{array}\right)\left(\begin{array}{c}
n-r \\
k
\end{array}\right)}{\left(\begin{array}{c}
n \\
k
\end{array}\right)} \mu_{i, r}^{n-k} & 0 \leq k \leq n-r \\
0 & k>n-r
\end{array},\right.
$$

where $\mu_{i, r}^{n-k}$ are given in (8).

Proof. From equation (10), it is clear that $\mathscr{U}_{n, r}^{(\gamma)}(u, v, w)$ has degree $\leq n-r$ in the variable $w$, thus

$$
a_{i j k}^{n, r}=0 \text { for } k>n-r .
$$

For $0 \leq k \leq n-r$, the remaining coefficients are determined by equating (10) and (17) as follows

$$
\sum_{i+j=n-k} a_{i j k}^{n, r} b_{i j k}^{n}(u, v, w)=(-1)^{k}\left(\begin{array}{c}
n+r+1 \\
k
\end{array}\right) b_{k}^{n-r}(w, u+v) \sum_{i=0}^{r} c(i) b_{i}^{r}(u, v) .
$$

Comparing powers of $w$ on both sides, we have

$$
\sum_{i=0}^{n-k} a_{i j k}^{n, r} \frac{n !}{i ! j ! k !} u^{i} v^{j}=(-1)^{k}\left(\begin{array}{c}
n+r+1 \\
k
\end{array}\right)\left(\begin{array}{c}
n-r \\
k
\end{array}\right)(u+v)^{n-r-k} \sum_{i=0}^{r} c(i) b_{i}^{r}(u, v) .
$$

The left hand side of the last equation can be written in the form

$$
\begin{aligned}
\sum_{i=0}^{n-k} a_{i j k}^{n, r} \frac{n !}{i ! j ! k !} u^{i} v^{j}=\sum_{i=0}^{n-k} a_{i j k}^{n, r} \frac{n !(n-k) !}{i !(n-k-i) ! k !(n-k) !} & u^{i} v^{j} \\
& =\sum_{i=0}^{n-k} a_{i j k}^{n, r}\left(\begin{array}{l}
n \\
k
\end{array}\right) b_{i}^{n-k}(u, v) .
\end{aligned}
$$


Therefore,

$$
\begin{aligned}
& \sum_{i=0}^{n-k} a_{i j k}^{n, r}\left(\begin{array}{c}
n \\
k
\end{array}\right) b_{i}^{n-k}(u, v) \\
& \quad=(-1)^{k}\left(\begin{array}{c}
n+r+1 \\
k
\end{array}\right)\left(\begin{array}{c}
n-r \\
k
\end{array}\right)(u+v)^{n-r-k} \sum_{i=0}^{r} c(i) b_{i}^{r}(u, v) .
\end{aligned}
$$

Using Lemma 3.2 with some binomial simplifications, we get

$$
\sum_{i=0}^{n-k} a_{i j k}^{n, r}\left(\begin{array}{l}
n \\
k
\end{array}\right) b_{i}^{n-k}(u, v)=(-1)^{k}\left(\begin{array}{c}
n+r+1 \\
k
\end{array}\right)\left(\begin{array}{c}
n-r \\
k
\end{array}\right) \sum_{i=0}^{r} \mu_{i, r}^{n-k} b_{i}^{n-k}(u, v)
$$

where $\mu_{i, r}^{n-k}$ are the coefficients resulting from writing Tchebyshev-II polynomial of degree $r$ in the Bernstein basis of degree $n-k$, as defined by expression (8). The result in (18) follows.

To derive a recurrence relation for the coefficients $a_{i j k}^{n, r}$ of $\mathscr{U}_{n, r}^{(\gamma)}(u, v, w)$, consider the generalized Bernstein polynomial of degree $n-1$,

$$
\begin{aligned}
b_{i j k}^{n-1}(u, v, w)= & \frac{(n-1) !}{i ! j ! k !} u^{i} v^{j} w^{k}(u+v+w) \\
= & \frac{(i+1)}{n} b_{i+1, j, k}^{n}(u, v, w)+\frac{(j+1)}{n} b_{i, j+1, k}^{n}(u, v, w) \\
& +\frac{(k+1)}{n} b_{i, j, k+1}^{n}(u, v, w) .
\end{aligned}
$$

By the construction of $\mathscr{U}_{n, r}^{(\gamma)}(u, v, w)$, we have

$$
\left\langle b_{i j k}^{n-1}(u, v, w), \mathscr{U}_{n, r}^{(\gamma)}(u, v, w)\right\rangle=0, \quad i+j+k=n-1 .
$$

Thus by Lemma 2.3

$$
(i+1) a_{i+1, j, k}^{n, r}+(j+1) a_{i, j+1, k}^{n, r}+(k+1) a_{i, j, k+1}^{n, r}=0 .
$$

From Theorem 4.1, $a_{i, n-i, 0}^{n, r}=\mu_{i, r}^{n}, i=0,1, \ldots, n$. Therefore, we can use (21) to generate $a_{i, j, k}^{n, r}$ recursively on $k$. 


\section{Acknowledgments}

The author thanks the referee for the comments and the suggestions.

\section{References}

[1] M. Abramowitz, I.A. Stegun (Eds.), Handbook of Mathematical Functions with Formulas, Graphs, and Mathematical Tables, 9th printing. New York: Dover (1972).

[2] M.-M. Derriennic, On multivariate approximation by Bernstein-type polynomials, J. Approx. Theory, 45, no. 2 (1985), 155-166.

[3] C.F. Dunkl, Y. Xu, Orthogonal Polynomials of Several Variables, Encyclopedia of Mathematics and its Applications, vol. 81, Cambridge University Press, Cambridge (2001).

[4] G. Farin, Curves and Surface for Computer Aided Geometric Design, 3rd ed., Computer Science and Scientific Computing, Academic Press, Massachusetts (1993).

[5] R.T. Farouki, T. N.T. Goodman, T. Sauer, Construction of orthogonal bases for polynomials in Bernstein form on triangular and simplex domains, Comput. Aided Geom. Design, 20, no. 4 (2003), 209-230.

[6] R.T. Farouki, V.T. Rajan, On the numerical condition of polynomials in Bernstein form, Comput. Aided Geom. Design, 4, no. 3 (1987), 191-216.

[7] J. Hoschek, D. Lasser, Fundamentals of Computer Aided Geometric Design, A K Peters, Massachusetts (1993).

[8] F.W.J. Olver, D.W. Lozier, R. F. Boisvert, and C. W. Clark, editors. NIST handbook of mathematical functions, Cambridge University Press, Cambridge (2010).

[9] A. Rababah, Distance for degree raising and reduction of triangular Bézier surfaces, J. Comput. Appl. Math., 158, no. 2 (2003), 233-241.

[10] A. Rababah, Transformation of ChebyshevBernstein polynomial basis, Comput. Methods Appl. Math., 3 (4) (2003), pp. 608-622.

[11] P.K. Suetin, Orthogonal Polynomials in Two Variables, Nauka, Moscow (1999). 
[12] G. Szegö, Orthogonal Polynomials, 4th ed., American Mathematical Society, Rhode Island (1975). 